Tôhoku Math. Journ.

$32(1980), 177-188$.

\title{
INTERVAL MAPS, FACTORS OF MAPS, AND CHAOS ${ }^{1}$
}

Dedicated to Professor Taro Yoshizawa on his sixtieth birthday

Joseph AUSLANDER AND JAmes A. YoRKe

(Received September 5, 1978)

\begin{abstract}
We investigate the dynamical properties of continuous maps of a compact metric space into itself. The notion of chaos is defined as the instability of all trajectories in a set together with the existence of a dense orbit. In particular we show that any map on an interval satisfying a generalized period three condition must have a nontrivial (uncountable) minimal set as well as "large" chaotic subsets. The nontrivial minimal sets are investigated by lifting to sequence spaces while the chaotic sets are investigated using "factors," projections of larger spaces onto smaller ones.
\end{abstract}

Introduction. There has been an increasing realization that the dynamics behind many physical processes are inherently chaotic. Just to cite an example, consider the fact that Landau [1] explained the transition to turbulence for flow past a solid object in terms that invoked an infinite number of degrees of freedom. Then Ruelle and Takens [2] showed that similar processes could lead to even more chaotic behavior while requiring only five degrees of freedom, i.e., five dimensions. Now it is recognized that only three degrees of freedom are required. Lorenz [3], Rossler [4, 5], Curry and Yorke [6] and Bowen [7] are just some of the papers which discuss the physical relevance of three dimensional chaotic flows or two dimensional chaotic diffeomorphisms. May [8] and $\mathrm{Li}$ and Yorke [9] emphasize the physical significance of continuous chaotic maps on the real line. A number of concepts have been introduced to describe the relevant phenomena. Most important is ergodic theory but this is particularly difficult to apply to particular situations; the simplest nonlinear dynamical process is the iteration of a quadratic map on an interval, $T(x)=r x(1-x)$, and yet even here the situation is unsatisfactory: The set of $r$ for which there is an absolutely continuous invariant probability measure has only recently been shown to be infinite (Pianigiani [10] and Jacobson and Sinai) yet it seems likely that this set

${ }^{1}$ This research was partially supported by National Science Foundation Grant MCS 7624432 
is uncountably infinite and may have positive measure.

A second approach is to discuss the flows in purely topological terms. Ruelle and Takens captured some of the flavor of turbulence with their introduction of the concept of "strange attractors," an attracting set which is compact and connected but is not a point, circle or $n$-torus. An alternative approach is to emphasize the chaotic nature of the dynamics rather than the shape of the set; hence the term "chaotic set" was introduced (Kaplan-Yorke [11, 12]; see also Li-Yorke [9]).

The notion of chaotic set arises as follows. In the investigation of systems with complex behavior, in particular turbulent fluids, it seems reasonable to assume first that the dynamical behavior occurs in some compact subset of phase space, that is, all limit sets of trajectories lie in a single compact set $X$. Secondly, we may assume the trajectories have sensitive dependence on initial data, that is, trajectories are Liapunov unstable. Finally some type of ergodicity is present, and at least there are dense orbits in the components of $X$. More generally chaotic sets seem to appear in many types of systems which have nothing to do with fluids.

In Yorke-Yorke [13] certain non-attracting chaotic sets are investigated. The topological concepts of chaotic behavior are combined with measure theory by Lasota, Pianigiani, and Yorke in $[14,15]$. The purpose of this paper is to further develop and investigate the topological concept of chaos. In particular one objective is to relate chaotic behavior in a large space to the chaotic behavior in a smaller, easier to analyze, space. For example, the solenoid is a space which can be obtained as follows: Let $T: X \rightarrow X$ where $X$ is the solid torus, and $T$ is an "into" diffeomorphism which stretches and double wraps the torus inside itself. The invariant set (the "solenoid") was the first stable "strange attractor" described, [2]. The map $T$ can be set up so that the angle $\theta$ around the torus is mapped to $2 \theta \bmod 2 \pi$. This map on the circle $\theta \mapsto 2 \theta$ is chaotic. This simpler space and map is called a factor of the original pair $(T, X)$ and the fact that the simpler space and map are chaotic imply in this case that the $(T, X)$ is also chaotic. This example motivates the investigation of chaos in factors.

Period three implies there are nontrivial minimal sets. A compact system is a pair $(X, \tau)$, where $X$ is a compact metric space, and $\tau: X \rightarrow X$ is continuous and onto. It is not assumed that $\tau$ is invertible.

If $x \in X$, the orbit of $x$ is the set $\gamma(x)=\left\{\tau^{j}(x) \mid j=0,1,2, \cdots\right\}$. The orbit $\gamma(x)$ is periodic if $\tau^{m}(x)=x$, for some $m>1$ (so $\gamma(x)=\{x, \tau(x), \cdots$, $\left.\tau^{m-1}(x)\right\}$ ), and $x$ is stationary if $\tau(x)=x$ (so $\gamma(x)=\{x\}$ ).

The omega limit set of $x$ is $\Omega(x)=\bigcap_{h \geqq 0} \overline{\gamma\left(\tau^{h}(x)\right)}$. Note that $y \in \Omega(x)$ 
if and only if there is a sequence of integers $n_{j} \rightarrow \infty$ such that $\tau^{n_{j}}(x) \rightarrow y$. Also, the orbit closure $\overline{\gamma(x)}=\gamma(x) \cup \Omega(x)$; this union is disjoint if and only if $x \notin \Omega(x)$.

An invariant set (for $\tau$ ) is a subset $M$ of $X$ such that $\tau(M) \subset M$. (Equivalently, $M$ is a union of orbits.) If $x \in X$, then $\gamma(x), \overline{\gamma(x)}$ and $\Omega(x)$ are invariant.

Sometimes (as in the case of interval maps) $\tau$ is defined on a set $Z$ for which $\tau(Z) \supset Z$. Our first lemma shows that, in this case, it is possible to "cut down" the domain of $\tau$ so as to obtain a compact system.

LEMMA 1. Let $X$ be a compact metric space, $Z$ a closed subset of $X$, and $\tau: Z \rightarrow X$ a continuous onto map. Let $Z^{*}=\bigcap_{k=0,1,2, \ldots} \tau^{-k}(Z)$. Then $Z^{*}$ is closed, non-empty, $\tau$-invariant, with $\tau\left(Z^{*}\right)=Z^{*}$. If $z \in Z$ such that $\tau(z) \in Z^{*}$, then $z \in Z^{*}$.

Proof. Most of the assertions are obvious. Note that $z \in Z^{*}$ if and only if $\gamma(z) \subset Z$. Also, $\gamma(\tau(z)) \subset \gamma(z)$, so $\tau(z) \in Z^{*}$ whenever $z \in Z^{*}$. If $z \in Z^{*}, \tau(x)=z$, for some $x \in Z$, and $\gamma(x)=\{x\} \cup \gamma(z) \subset Z$, so $x \in Z^{*}$. Hence $\tau\left(Z^{*}\right)=Z^{*}$.

Using the notation of Lemma 1 , suppose that $A$ and $B$ are closed non-empty subsets of $Z$ with $Z=A \cup B, \tau(A) \supset B, \tau(B) \supset Z$. Set $A^{*}=$ $A \cap Z^{*}, B^{*}=B \cap Z^{*}$, so $Z^{*}=A^{*} \cup B^{*}$. If $x \in Z^{*}$, then $x=\tau(b)$, for some $b \in B$, so $b \in Z^{*} \cap B=B^{*}$, and $B^{*} \neq \varnothing$. Also $b=\tau(a)$, for some $a \in A$, and $a \in Z^{*} \cap A=A^{*}$, and $A^{*} \neq \varnothing$. The above shows $\tau\left(A^{*}\right) \supset B^{*}$ and $\tau\left(B^{*}\right) \supset Z^{*}$. Since $\tau\left(B^{*}\right) \subset \tau\left(Z^{*}\right)=Z^{*}$, we actually have $\tau\left(B^{*}\right)=Z^{*}$.

We remark that $A^{*}$ and $B^{*}$ are both infinite sets, unless $A^{*}=B^{*}=$ $Z^{*}$. For, since $\tau\left(A^{*}\right) \supset B^{*}, \tau\left(B^{*}\right)=A^{*} \cup B^{*}$, then $A^{*}$ is finite if and only if $B^{*}$ is finite. Letting $|\cdot|$ denote the cardinal number, suppose $\left|A^{*}\right|=n$, $\left|B^{*}\right|=m, \quad\left|A^{*} \cap B^{*}\right|=k$ (with $m, n, k<\infty$ ). Since $\tau\left(A^{*}\right) \supset B^{*}$, and $\tau\left(B^{*}\right)=A^{*} \cup B^{*}$, we have $n \geqq m, m+n-k \leqq m$ and therefore $n \leqq k$. But $k \leqq m \leqq n$, so $k=m=n$. That is $\left|A^{*} \cap B^{*}\right|=n=\left|A^{*}\right|=\left|B^{*}\right|$, and $A^{*}=B^{*}$.

We apply these considerations to a compact interval $Z=[a, c]$ on the real line. Let $\tau: Z \rightarrow \boldsymbol{R}$ be continuous and suppose there is a $b \in Z$ with $a<b<c$ such that $\tau(a)=b, \tau(b)=c, \tau(c) \leqq a$. This "generalized period three" condition was studied in [4], where it was shown that it implies the existence of points of every period $k \geqq 1$. We may suppose further that if $a<x<b$, then $\tau(x)>b$ (if not, let $a^{*}=\sup [y \in[a, b] \mid \tau(y)=b]$ and replace $a$ by $\left.a^{*}\right)$.

If $A=[a, b], B=[b, c]$, then $\tau(A) \supset B, \tau(B) \supset Z$, and the previous 
discussion applies with $X=\tau(Z)$. Note also that $\tau(A) \cap A=\{b\}$, so that $\tau\left(A^{*}\right)=B^{*}$.

To study $\tau$ further we introduce the one sided shift. Let $\Sigma=\{0,1\}^{v}$, the collection of infinite one sided sequences of 0 's and 1 's, with the product topology. A metric for $\Sigma$ is given by

$$
d\left(\left(\omega_{0}, \omega_{1}, \cdots\right),\left(\omega_{0}^{\prime}, \omega_{1}^{\prime}, \cdots\right)\right)=\sum_{j=0}^{\infty}\left|\omega_{j}-\omega_{j}^{\prime}\right| 2^{-j} .
$$

Let $\sigma: \Sigma \rightarrow \Sigma$ be the shift transformation $\sigma\left(\omega_{0}, \omega_{1}, \cdots\right)=\left(\omega_{1}, \omega_{2}, \cdots\right)$. Then $\Sigma$ is a compact metric space and $\sigma$ is a continuous onto two to one map. The pair $(\Sigma, \sigma)$ is called the one sided shift on the symbols 0 and 1.

From now on, we use $A^{*}$ and $B^{*}$ (instead of 0 and 1 ) as the symbols in $\Sigma$, and we omit parentheses and commas in writing the points of $\Sigma$. Thus $A^{*}$ and $B^{*}$ will have two roles: subsets of $Z=[a, c]$, and symbols for $\Sigma$. It will be clear from the context which meaning is intended.

The point $\omega=\omega_{0} \omega_{1} \cdots \in \Sigma$ will be called admissible if it contains no 2 block of the form $A^{*} A^{*}$ (that is, if $\omega_{j}=A^{*}$, then $\omega_{j+1}=B^{*}$ ). The subset $\Sigma^{*}$ of admissible points is a closed $\sigma$-invariant subset of $\Sigma$. We show that every point of $Z^{*}$ defines an admissible sequence, and that every admissible sequence gives rise to a closed non-empty subset of $Z^{*}$. In fact, there is a natural map $\pi: Z^{*} \rightarrow \Sigma^{*}$ defined by $\pi(z)=\omega=$ $\omega_{0} \omega_{1} \omega_{2} \cdots$ where $\omega_{i}=A^{*}$ or $B^{*}$ according as $\tau^{i}(z) \in A^{*}$ or $B^{*}$. (If $b \in Z^{*}$ and $\tau^{i}(z)=b$, let $\omega_{i}=B^{*}$. This choice, and the standing assumption that $\tau(x)>b$ for $a<x<b$, guarantee that $\omega$ is admissible.)

Theorem 1. (i ) The map $\pi: Z^{*} \rightarrow Z^{*}$ is equivariant $(\pi \tau=\sigma \pi)$ and onto.

(ii) $\pi$ is continuous at all points of $Z^{*} \backslash P$, where $P=\{b\} \cup\left[z \in Z^{*}\right.$ $\tau^{k}(z)=b$ and $\tau^{k-1}(z) \in B^{*}$, for some $\left.k>0\right]$. (So, if $\tau(c)<a$, then $P=\varnothing$, and $\pi$ is continuous on $Z^{*}$.)

(iii) If $z \in Z^{*}, \pi(z)=\omega$, and $\overline{\gamma(z)}$ contains a periodic point, then $\overline{\gamma(\omega)}$ contains a periodic point.

(iv) If $\omega \in \Sigma^{*}$ is periodic, then $\pi^{-1}(\omega)$ contains a periodic point.

Proof. Equivariance and continuity on $Z^{*} \backslash P$ are obvious. If $\omega \in \Sigma^{*}$, $\omega=\omega_{0} \omega_{1} \cdots$, let $C_{n}(\omega)=\omega_{0} \cap \tau^{-1}\left(\omega_{1}\right) \cap \cdots \cap \tau^{-n}\left(\omega_{n}\right)$. Then each $C_{n}(\omega)$ is closed and non-empty, and also $C_{0}(\omega) \supset C_{1}(\omega) \cdots$. Thus $\pi^{-1}(\omega)=$ $\bigcap_{n=1,2, \ldots} C_{n}(\omega)$ is closed and non-empty, so $\pi$ is onto. Suppose $z^{\prime} \in \overline{\gamma(z)}$ is periodic. If $z^{\prime} \notin P, \pi$ is continuous at $z^{\prime}$ and it follows easily that $\omega^{\prime}=\pi\left(z^{\prime}\right) \in \overline{\gamma(\omega)}$ and $\omega^{\prime}$ is periodic. If $z^{\prime} \in P$ with $z^{\prime}$ periodic, then $z^{\prime}=b$. 
But then $a=\tau(b) \in \overline{\gamma(z)}$, and since $\pi$ is continuous at $a$, we again have $\pi(a) \in \overline{\gamma(\omega)}$ is periodic. Let $\omega \in \Sigma^{*}$ be periodic, $\omega=\omega_{0} \omega_{1} \cdots \omega_{k-1} \omega_{0} \omega_{1} \cdots$ $\omega_{k-1} \cdots$. Let $\lambda_{i}=A$ or $B$ according as $\omega_{i}=A^{*}$ or $B^{*}(i=0,1, \cdots, k-1)$. Choose $x \in \lambda_{0}$ such that $\tau^{i}(x) \in \lambda_{i}(i=0,1, \cdots, k-1)$ and such that $\tau^{k}(x)=x$ (see [9], Lemmas 0 and 2). Then certainly $x \in Z^{*}, x$ is periodic, and $\pi(x)=\omega$.

Note that (iv) is a partial converse of (iii). An interesting question is whether the complete converse of (iii) is valid. Namely if $z \in Z^{*}$, $\pi(z)=\omega$, and $\overline{\gamma(\omega)}$ contains a periodic point in $\Sigma^{*}$, does $\overline{\gamma(z)}$ contain a periodic point in $Z^{*}$ ?

Recall that a minimal set is a non-empty closed invariant set, which contains no proper non-empty closed invariant subsets. Equivalently, the non-empty set $M$ is minimal if, for each $x \in M$, the orbit closure $\overline{\gamma(x)}=M$. An argument using Zorn's lemma shows that if $(X, \tau)$ is a compact system any non-empty closed invariant subset of $X$ contains minimal subsets. Of course, stationary and periodic orbits are minimal sets. We will call a minimal set $M$ non-trivial if it is not one of thesethat is, if $M$ is infinite.

Corollary 1. $Z^{*}$ contains non-trivial minimal sets.

Proof. Let $\omega \in \Sigma^{*}$ such that $\overline{\gamma(\omega)}$ contains no periodic points (for example, let $\overline{\gamma(\omega)}$ be a non-trivial minimal set in $\left.\Sigma^{*}\right)$, and let $x \in \pi^{-1}(\omega)$. Since $\overline{\gamma(x)}$ is closed and invariant, it contains minimal sets. Now $\overline{\gamma(x)}$ contains no periodic points (Theorem 1 , (iii)), so any minimal set in $\overline{\gamma(x)}$ must be non-trivial.

An explicit example of a non-trivial minimal set in $\Sigma^{*}$ will be given later.

Sensitive dependence on initial conditions (transitivity and chaos). Let $(X, \tau)$ be an arbitrary compact system. The point $x \in X$ is said to be stable if, for any $\varepsilon>0$, there is a $\delta>0$ such that $d\left(x, x^{\prime}\right)<\delta$ implies $d\left(\tau^{n}(x), \tau^{n}\left(x^{\prime}\right)\right)<\varepsilon$, for $n=0,1,2, \cdots$. (We also say $\tau$ is stable at $x$.) Thus $x$ is not stable (unstable) if and only if there is an $\varepsilon>0$, and a sequence $\left\{x_{j}\right\}$ in $X$ and $\left\{n_{j}\right\}$ of positive integers such that $x_{j} \rightarrow x$ and $d\left(\tau^{n_{j}}(x), \tau^{n_{j}}\left(x_{j}\right)\right) \geqq \varepsilon$. (The continuity of $\tau$ forces $n_{j} \rightarrow \infty$.) In this case, we say that $x$ is $\varepsilon$ unstable.

Lemma 2. Let $K_{s}$ denote the set of $\varepsilon$ unstable points. Then $\bar{K}_{\varepsilon} \subset K_{\varepsilon / 2}$.

Proof. Let $\left\{x_{j}\right\}$ be a sequence in $K_{\varepsilon}$, and let $x_{j} \rightarrow x$. Since each $x_{j}$ is $\varepsilon$ unstable, there is a sequence $\left\{x_{j}^{\prime}\right\}$ with $d\left(x_{j}, x_{j}^{\prime}\right) \rightarrow 0$ and $d\left(\tau^{h}\left(x_{j}\right)\right.$, 
$\left.\tau^{h_{j}}\left(x_{j}^{\prime}\right)\right) \geqq \varepsilon$, for some sequence $\left\{h_{j}\right\}$ of positive integers. If $d\left(\tau^{h_{j}}(x), \tau^{h_{j}}\left(x_{j}\right)\right) \geqq$ $\varepsilon / 2$, then certainly $x$ is $\varepsilon / 2$ unstable. If not, then $d\left(\tau^{h_{j}}(x), \tau^{h_{j}}\left(x_{j}^{\prime}\right)\right) \geqq \varepsilon / 2$ (otherwise $d\left(\tau^{h_{j}}\left(x_{j}\right), \tau^{h_{j}}\left(x_{j}^{\prime}\right)\right)<\varepsilon$, contradicting the above). Since $x_{j} \rightarrow x$ and $x_{j}^{\prime} \rightarrow x, \varepsilon / 2$ instability of $x$ is proved.

Corollary 2. Let $(X, \tau)$ be a compact system.

(i ) If $x \in X$ is $\varepsilon$ unstable, then every point of $\overline{\gamma(x)}$ is $\varepsilon / 2$ unstable.

(ii) If $(X, \tau)$ is minimal and contains an unstable point, then there is an $\varepsilon>0$ such that every point is $\varepsilon$ unstable.

Proof. If $x$ is $\varepsilon$ unstable, so is $\tau(x)$. Thus (i) follows from Lemma 2 , and (ii) follows from (i).

The compact system $(X, \tau)$ is said to be chaotic if every point is unstable, and $X$ contains a dense orbit, ([2]). (It follows that a minimal set which contains an unstable point is chaotic.) We now develop an alternate characterization of chaos in terms of topological transitivity. We say that $(X, \tau)$ is topologically transitive if, for every non-empty open set $U$, the set $U^{*}=\bigcup_{k=1,2, \ldots} \tau^{-k}(U)$ is dense in $X$.

Lemma 3. Let $(X, \tau)$ be a compact system. Then the following are equivalent:

(i ) $(X, \tau)$ is topologically transitive.

(ii) There is an $x \in X$ with a dense orbit.

(iii) There is an $x \in X$ with $\Omega(x)=X$.

Proof. If $\Omega(x)=X$, then certainly $\overline{\gamma(x)}=X$. Suppose there is an $x \in X$ with $\overline{\gamma(x)}=X$. Let $x^{\prime} \in X$ with $\tau\left(x^{\prime}\right)=x$. Now $x^{\prime} \in \overline{\gamma(x)}=\gamma(x) \cup$ $\Omega(x)$. If $x^{\prime} \in \gamma(x)$, then $x=\tau\left(x^{\prime}\right) \in \gamma(x)$ and $\gamma(x)=\overline{\gamma(x)}=\Omega(x)=X$ is a finite set. If $x^{\prime} \in \Omega(x)$, then $x=\tau\left(x^{\prime}\right) \in \Omega(x)$, and, since $\Omega(x)$ is always closed and invariant, $\overline{\gamma(x)} \subset \Omega(x)$, so $\Omega(x)=X$.

Suppose $(X, \tau)$ is topologically transitive. Let $\left\{U_{1}, U_{2}, \cdots\right\}$ be a countable base for the open sets of $X$, and, if $j$ and $N$ are positive integers, let $U_{j}^{(N)}=\bigcup_{k \geqq N} \tau^{-k}\left(U_{j}\right)$. Then (since $\tau^{-N}\left(U_{j}\right) \neq \varnothing$ and open), $U_{j}^{(N)}$ is open and dense. By the Baire category theorem there is an $x \in \bigcap_{j>0, N>0} U_{j}^{(N)}$. Thus, for every pair of positive integers $j$ and $N, x \in U_{j}^{(N)}$, so $\tau^{k}(x) \in U_{j}$, for some $k \geqq N$. Hence $\Omega(x) \cap \bar{U}_{j} \neq \varnothing$, and it follows that $\Omega(x)=X$.

Finally, suppose $\Omega(x)=X$, for some $x$, and let $U$ and $W$ be open sets in $X$. We show $U^{*} \cap W \neq \varnothing$ (where, as above $U^{*}=\bigcup \tau^{-k}(U)$ ). Let $k>0$ such that $\tau^{k}(x) \in W$ and let $l>0$ such that $\tau^{k+l}(x) \in U$, so $\tau^{k}(x) \in W \cap \tau^{-l}(U) \subset W \cap U^{*}$.

Note that the preceding proof shows that if there is a point with a dense orbit, then there is a residual set of such points. A point with 
a dense orbit will be called a transitive point.

Trivial examples (e.g., an orbit tending to a stationary point) show that if $\tau: X \rightarrow X$ is not onto, the existence of a dense orbit does not imply topological transitivity. However, if $x$ is unstable, and has a dense orbit, then $\Omega(x)=X$ (in which case $\tau$ is necessarily onto). Since $x$ is unstable there is a sequence $y_{j} \rightarrow x$ with $d\left(\tau^{n_{j}}(x), \tau^{n_{j}}\left(y_{j}\right)\right) \geqq \varepsilon>0$, for some $\left\{n_{j}\right\}$. This inequality implies that $y_{j} \neq x$ and we may assume all the $y_{j}$ are distinct. If $y_{j} \in \gamma(x), y_{j}=\tau^{n_{j}}(x)$, and $n_{j} \rightarrow \infty$. Thus $x \in \Omega(x)$. If $y_{j} \notin$ $\gamma(x)$, then, since $y_{j} \in \overline{\gamma(x)}=\gamma(x) \cup \Omega(x)$, so $y_{j} \in \Omega(x)$, and, since $\Omega(x)$ is closed, again $x \in \Omega(x)$. Then $X=\overline{\gamma(x)} \subset \Omega(x)$, so $\Omega(x)=X$.

Thus we may define chaos as "topological transitivity plus pointwise instability." This formulation (which by Lemma 3 is equivalent to the definition) has the advantage of homogeneity - it is not necessary to single out a point with a dense orbit.

Chaotic factors. We now consider the problem of "lifting" chaos via homomorphisms. If $(X, \tau)$ and $(Y, \varphi)$ are compact systems, a homomorphism of $(X, \tau)$ to $(Y, \varphi)$ is a continuous onto map $f: X \rightarrow Y$ such that $f \tau=\varphi f . \quad(Y$ is called a homomorphic image or factor of $X$.) The map $\pi: Z^{*} \backslash P \rightarrow \Sigma^{*}$ considered earlier is an example.

LEMMA 4. Let $(X, \tau)$ and $(Y, \varphi)$ be compact systems and $f: X \rightarrow Y$ a homomorphism. Let $x \in X$ be a stable point, and suppose that $f$ is open at $x$. Then $y=f(x)$ is stable.

Proof. If $y$ is unstable, there are sequences $y_{n} \rightarrow y$ and $\left\{k_{n}\right\}$ such that $d\left(\varphi^{k_{n}}(y), \varphi^{k_{n}}\left(y_{n}\right)\right) \geqq \varepsilon>0$. By openness of $f$ at $x$, there is a sequence $x_{n} \rightarrow x$ with $f\left(x_{n}\right)=y_{n}$. Since $x$ is stable, $d\left(\tau^{k_{n}}(x), \tau^{k_{n}}\left(x_{n}\right)\right) \rightarrow 0$, so $d\left(f\left(\tau^{k_{n}}(x)\right), f\left(\tau^{k_{n}}\left(x_{n}\right)\right)\right) \rightarrow 0$. That is $d\left(\varphi^{k_{n}}(y), \varphi^{k_{n}}\left(y_{n}\right)\right) \rightarrow 0$, which is a contradiction.

THEOREM 2. Let $(X, \tau)$ and $(Y, \varphi)$ be topologically transitive compact systems, with $(Y, \varphi)$ chaotic. Let $f: X \rightarrow Y$ be a homomorphism which is open at a transitive point $x$. Then $(X, \tau)$ is chaotic.

Proof. By Lemma $4, x$ is unstable. Since $x$ is a transitive point, it follows (Corollary 2), that all points of $X$ are stable.

A natural question is whether the openness hypotheses on $f$ can be dispensed with. Theorem 2 does apply to "skew product" systems $\varphi: X \times Y \rightarrow X \times Y$, of the form $\varphi(x, y)=(\tau(x), \psi(x, y))$, where $(X, \tau)$ is chaotic, and $\psi: X \times Y \rightarrow Y$ is chosen so that $\varphi$ is topologically transitive.

THEOREM 3. Let $(X, \tau)$ and $(Y, \varphi)$ be compact systems and let 
$f: X \rightarrow Y$ be a homomorphism. Suppose $(Y, \varphi)$ is chaotic. Then there is a closed $\tau$-invariant subset $X_{0}$ of $X$ such that $f\left(X_{0}\right)=Y$ and such that the subsystem $\left(X_{0}, \tau\right)$ is chaotic.

Proof. Using Zorn's lemma and the compactness of $X$ we can find a closed $\tau$ invariant subset $X_{0}$ of $X$ such that $f\left(X_{0}\right)=Y$, and such that $X_{0}$ is minimal with respect to these properties. Let $y \in Y$ be a transitive point, and let $x_{0} \in X_{0}$ such that $f\left(x_{0}\right)=y$. It follows easily that $f\left(\Omega\left(x_{0}\right)\right)=\Omega(y)=Y$, and since $\Omega\left(x_{0}\right)$ is a closed $\tau$ invariant subset of $X_{0}$, the defining minimality property of $X_{0}$ implies that $\Omega\left(x_{0}\right)=X_{0}$.

Since $(Y, \varphi)$ is chaotic, there are sequences $y_{n} \rightarrow y$, and integers $\left\{k_{n}\right\}$ such that $d\left(\varphi^{k_{n}}(y), \varphi^{k_{n}}\left(y_{n}\right)\right) \geqq \varepsilon>0$. Let $x_{n} \in X_{0}$ with $f\left(x_{n}\right)=y_{n}$, and choose a convergent subsequence of $\left\{x_{n}\right\}$. Still call the subsequence $\left\{x_{n}\right\}$ and let $x_{n} \rightarrow x_{0} \in X_{0}$. Then $f\left(x_{0}\right)=y_{0}$. Now, if $d\left(\tau^{k_{n}}\left(x_{0}\right), \tau^{k_{n}}\left(x_{n}\right)\right) \rightarrow 0$, then $d\left(\varphi^{k_{n}}\left(y_{0}\right), \varphi^{k_{n}}\left(y_{n}\right)\right)=d\left(\varphi^{k_{n}}\left(f\left(x_{0}\right)\right), \varphi^{k_{n}}\left(f\left(x_{n}\right)\right)\right)=d\left(f\left(\tau^{k_{n}}\left(x_{0}\right)\right), f\left(\tau^{k_{n}}\left(x_{n}\right)\right)\right) \rightarrow 0$, by continuity of $f$. This contradicts $d\left(\phi^{k_{n}}\left(y_{0}\right), \varphi^{k_{n}}\left(y_{n}\right)\right) \geqq \varepsilon>0$ so there is an $\bar{\varepsilon}>0$ such that $d\left(\tau^{k_{n}}\left(x_{0}\right), \tau^{k_{n}}\left(x_{n}\right)\right) \geqq \bar{\varepsilon}$. Hence $x_{0}$ is an unstable point. By Corollary 2, every point of $\Omega\left(x_{0}\right)=X_{0}$ is unstable, so $\left(X_{0}, \tau\right)$ is chaotic.

Corollary 3. Let $(X, \tau)$ and $(Y, \varphi)$ be minimal sets, with $Y$ a factor of $X$, and $(Y, \varphi)$ chaotic. Then $(X, \tau)$ is chaotic.

One way of showing that a system is chaotic (or has chaotic subsets) is to find a chaotic factor and apply Theorem 2 or Theorem 3 . In particular, the space $\Sigma^{*}$ of admissible sequences is chaotic. For, if $\omega^{*} \in \Sigma^{*}$ is a sequence which contains all finite blocks which do not contain $A^{*} A^{*}$ (so it necessarily contains every such block infinitely often) then $\Omega\left(\omega^{*}\right)=\Sigma^{*}$. Moreover, every point of $\Sigma^{*}$ is unstable; in fact $\left(\Sigma^{*}, \sigma\right)$ is expansive: there is an $\alpha>0$ such that if $\omega \neq \omega^{\prime}$, then $d\left(\sigma^{m}(\omega), \sigma^{m}\left(\omega^{\prime}\right)\right)>\alpha$, for some $m \geqq 0$. It follows that any infinite (hence, self dense) topologically transitive subset of $\Sigma^{*}$ is chaotic.

Applying these considerations to $Z=[a, c]$, recall that if $\tau(c)<a$, then $\Sigma^{*}$ is a factor of $Z^{*}$, so (Theorem 3 ) there is a closed $\tau$ invariant subset $Z_{0}^{*}$ of $Z^{*}$ such that $\pi\left(Z_{0}^{*}\right)=\Sigma^{*}$, and $\left(Z_{0}^{*}, \tau\right)$ is chaotic. Even if $\tau(c)=a$, any infinite minimal set $\overline{\gamma(\omega)}$ in $\Sigma^{*}$ has a minimal preimage $\overline{\gamma(x)}$ in $Z^{*}$ and (Corollary 3$), \overline{\gamma(x)}$ is chaotic.

There is an extensive literature on shift space and its minimal subsets. Most of the literature is concerned with the bilateral (or two sided) shift - the space of doubly infinite sequences $\omega=\cdots \omega_{-1} \omega_{0} \omega_{1} \cdots$ $\left(\omega_{i}=0\right.$ or 1$)$, where the shift transformation, defined by $\sigma(\omega)=\omega^{\prime}$, $\omega_{j}^{\prime}=\omega_{j+1}$, is a homeomorphism. However, it is easy to check that if 
$\omega=\cdots \omega_{-1} \omega_{0} \omega_{1} \cdots$ has a minimal orbit closure in the bilateral shift then $\omega^{*}=\omega_{0} \omega_{1} \cdots$ has a minimal orbit closure in the one sided shift, and that every minimal set in the one sided shift is obtained in this manner, as a "restriction" of a (unique) minimal set in the bilateral shift.

A class of examples of minimal sets in $\Sigma^{*}$ are the Sturmian minimal sets, [1]. To define them, consider a circle $C$ of circumference 1 , and let $\alpha$ be an irrational number with $0<\alpha<1 / 2$. Let $[a, b]$ be a closed interval of length $\alpha$ on $C$ and let $\rho: C \rightarrow C$ be the counterclockwise rotation by $\alpha$. If $p \in C$, define $\omega_{p}^{+}$and $\omega_{p}^{-}$in $\Sigma^{*}$ by

$$
\omega_{p}^{+}(i)=\left\{\begin{array}{lll}
A^{*} & \text { if } & \rho^{i}(p) \in(a, b] \\
B^{*} & \text { if } & \rho^{i}(p) \notin(a, b]
\end{array}\right.
$$

and

$$
\omega_{p}^{-}(i)=\left\{\begin{array}{lll}
A^{*} & \text { if } & \rho^{i}(p) \in[a, b) \\
B^{*} & \text { if } & \rho^{i}(p) \notin[a, b)
\end{array} .\right.
$$

(Note that $\omega_{p}^{+}=\omega_{p}^{-}$if and only if neither $a$ nor $b$ are in the $\rho$ orbit of $p$.) Then $\omega_{p}^{+}$and $\omega_{p}^{-}$are in $\Sigma^{*}$, and $\Gamma_{\alpha}=\left\{\omega_{p}^{+} \mid p \in C\right\} \cup\left\{\omega_{p}^{-} \mid p \in C\right\}$ is minimal under $\sigma$.

It is intriguing that a space can be chaotic and can also have many chaotic subsets, and the shift space is an example with its many minimal sets. Any subshift is also a chaotic subset.

Pointwise stable minimal sets have one-to-one maps. We return again to compact systems in general, and consider the consequences of pointwise stability (all points stable). For minimal sets, pointwise stability is the opposite of chaos. The straightforward proof of the following result is omitted.

Lemma 5. (i) If $(X, \tau)$ is pointwise stable, it is uniformly stable. (That is, the collection $\left\{\tau^{n} \mid n=1,2, \cdots\right\}$ is a uniformly equicontinuous family.)

(ii) If $(X, \tau)$ is pointwise stable, then every factor is pointwise stable.

THEOREM 4. Suppose $(X, \tau)$ is pointwise stable and topologically transitive. Then $(X, \tau)$ is a minimal set, and $\tau$ is a homeomorphism.

Proof. Let $x \in X$ be a transitive point, and let $y \in X$. Then there are sequences $\left\{k_{i}\right\}$ and $\left\{l_{i}\right\}$ of positive integers such that $\tau^{k_{i}}(x) \rightarrow y$, and $\tau^{k_{i}+l_{i}}(x) \rightarrow x$. Since $y$ is a stable point $d\left(\tau^{l_{i}+k_{i}}(x), \tau^{l_{i}}(y)\right)=d\left(\tau^{l_{i}}\left(\tau^{k_{i}}(x)\right), \tau^{l_{i}}(y)\right) \rightarrow 0$, so $d\left(x, \tau^{l_{i}}(y)\right) \rightarrow 0$, and $x \in \overline{\gamma(y)}$. Thus $y$ is a transitive point. Since $y$ is 
arbitrary, $(X, \tau)$ is minimal.

To show that $\tau$ is a homeomorphism, consider the space $X^{X}$, the set of all maps (not necessarily continuous) of $X$ to itself, provided with the product topology (the topology of pointwise convergence). Let $G$ be the closure of the set $\left\{\tau^{n} \mid n=1,2, \cdots\right\}$ in $X^{X}$. Since $\left\{\tau^{n} \mid n=1,2, \cdots\right\}$ is an equicontinuous family, it follows easily that all elements of $G$ are continuous. If $x, y \in X$, there is a $\xi \in G$ such that $\xi(y)=x$ (since $\gamma(y)$ is dense in $X$ and $X^{X}$ is compact). Moreover, $\xi \tau=\tau \xi$. (For, let $\left\{\tau^{n^{i}}\right\}$ be a net such that $\tau^{n_{i}} \rightarrow \xi$. Then $\tau^{n_{i+1}}=\tau \tau^{n_{i}} \rightarrow \tau \xi$ and $\tau^{n^{+1}}=\tau^{n_{i}} \rightarrow \xi \tau$.) Now, let $x \in X$, and let $y=\tau(x)$. Let $\xi \in G$ such that $\xi(y)=x$. Then $\tau \xi(x)=\xi \tau(x)=\xi(y)=x$. Also $\xi \tau^{k}=$ $\tau^{k} \xi$, for all $k>0$, and $\xi \tau\left(\tau^{k}(x)\right)=\tau^{k}(\xi \tau(x))=\tau^{k}(x)$; similarly, $\tau \xi\left(\tau^{k}(x)\right)=$ $\tau^{k}(x)$. Hence $\tau \xi=\xi \tau$ is the identity on $\gamma(x)$, and, since $\overline{\gamma(x)}=X, \tau \xi=$ $\xi \tau$ is the identity map. Therefore $\tau$ is invertible.

Corollary 4. Let $(X, \tau)$ be a nonchaotic minimal set. Then $\tau$ is a homeomorphism.

Proof. Since $(X, \tau)$ is not chaotic, it contains a stable point. By Corollary 2, all points of $X$ are stable. Thus Theorem 4 applies, and $\tau$ is a homeomorphism.

An example of a (necessarily chaotic) minimal set with respect to a continuous map which is not a homeomorphism is obtained as follows. Let $h: X \rightarrow X$ be a minimal homeomorphism which contains asymptotic points - that is, $x \neq y$ such that $d\left(h^{n}(x), h^{n}(y)\right) \rightarrow 0$, as $n \rightarrow \infty$. (The Sturmian minimal sets discussed earlier are an example.) Consider a fixed pair of asymptotic points $x$ and $y(x \neq y)$. Define an equivalence relation $\sim$ on $X$ by declaring $h^{n}(x) \sim h^{n}(y),(n=1,2, \cdots)$. Since $x$ and $y$ are asymptotic the quotient space $\widetilde{X}=X / \sim$ is compact Hausdorff, hence metrizable. Moreover, $h$ induces a continuous map $\tilde{h}: \widetilde{X} \rightarrow \widetilde{X} .(\tilde{X}, \tilde{h})$ is minimal, since it is a factor of the minimal set $(X, h)$. If [ ] denotes equivalence class, then $[x] \neq[y]$, but $\widetilde{h}([x])=[h(x)]=[h(y)]=\widetilde{h}([y])$, so $\tilde{h}$ is not a homeomorphism.

As we have noted, a minimal set is either chaotic or pointwise stable. We do not know whether the same phenomenon holds for any topologically transitive system $(X, \tau)$, even when $\tau$ is a homeomorphism. If, as seems likely, a counterexample exists, all transitive points would have to be stable (Corollary 2 again). An unsolved related question, relevant to Theorem 3, is: must a topologically transitive system which has a chaotic factor be chaotic? An example to the contrary would also serve as a counterexample for the previous question (Corollary 2 and Lemma 5). 
The construction of such examples appears to be rather difficult. If we again consider $\tau:[a, c] \rightarrow \boldsymbol{R}$ with $\tau(c)<a$, then no topologically transitive subset $Z^{\prime}$ of $Z^{*}$ with $\pi\left(Z^{\prime}\right)=\Sigma^{*}$ will provide an example. That is, any such $Z^{\prime}$ is chaotic. For, since $\tau(c)<a, b \notin Z^{*}$ and since $Z^{*}$ is closed, there is an $\varepsilon>0$ such that $\left|z^{\prime}-b\right|>\varepsilon$, for all $z^{\prime} \in Z^{\prime}$. Now, suppose $z \in Z^{*}$ is a stable point. Let $\delta>0$ correspond to the $\varepsilon$ above in the definition of stability of $z$. Then if $z^{\prime} \in Z^{*}$ with $\left|z-z^{\prime}\right|<\delta$, then $\pi(z)=\pi\left(z^{\prime}\right)$. (If not, there would be an integer $k$ such that, say $\tau^{k}(z) \in A^{*}$ and $\tau^{k}\left(z^{\prime}\right) \in B^{*}$. Therefore, $\left|\tau^{k}(z)-\tau^{k}\left(z^{\prime}\right)\right| \geqq \varepsilon$, contradicting the choice of $\delta$ above.) Now, suppose in addition that $z$ is a transitive point of $Z^{\prime}$. In particular, $z \in \Omega(z)$. Then there is an $m$ such that $\left|z-\tau^{m}(z)\right|<\delta$, so $\pi(z)=\pi\left(\tau^{m}(z)\right)$. If $\omega=\pi(z) \in \Sigma^{*}$, then $\omega=\sigma^{m}(\omega)$, so $\omega$ is a periodic point. This is a contradiction, since a transitive point of $Z^{\prime}$ must be mapped to a transitive point of $\Sigma^{*}$ by a homomorphism.

\section{REFERENCES}

[1] L. D. Landau and E. M. Lifschitz, Fluid Mechanics, Pergamon Press, Oxford, 1959. See also L. Landau, C. R. Acad. Sci. U.R.S.S. 44 (1944), 311-314.

[2] D. Ruelle and F. Takens, On the nature of turbulence, Comm. Math. Phys. 20 (1971), 167-192; 23 (1971), 343-344.

[3] E. LoREnz, Deterministic nonperiodic flow, J. Atmos. Sci. 20 (1963), 130-141.

[4] O. E. Rossler, An equation for continuous chaos, Physics Letters 57A, no. 5 (19 July 1976), 397-398.

[5] O. E. Rossler, Different types of chaos in two simple differential equations, Z. Naturforsch 31a (1976), 1664-1670.

[6] J. CURRY AND J. A. YoRke, A transition from Hopf bifurcation to chaos: computer experiments with maps in $R^{2}$, in Proceedings of the NSF Regional Conference in Fargo, N. D., June, 1977, to appear.

[7] R. BowEN, A model for Couette flow data, in Springer-Verlag Lecture Notes \#615: Turbulence Seminar, 1977.

[8] R. M. MAY, Biological population with nonoverlapping generations: stable points, stable cycles and choas, Science 186 (1974), 645-647.

[9] T. Y. Li AND J. A. Yonke, Period three implies chaos, Amer. Math. Monthly 82 (1975), 985-992.

[10] G. Pianigiani, Absolutely continuous invariant measures for the process $x_{n+1}=A x_{n}\left(1-x_{n}\right)$, preprint.

[11] J. Kaplan and J. A. YoRke, Preturbulence: a regime observed in a fluid flow model of Lorenz, preprint.

[12] J. Kaplan AND J. A. Yorke, The onset of chaos in a fluid flow model of Lorenz, Proceedings of the New York Acad. of Sciences Meeting Bifurcation, to appear.

[13] J. A. Yorke AND E. D. YoRKE, Metastable chaos: The transition to sustained chaotic behavior in the Lorenz model, preprint.

[14] A. Lasota and J. A. YoRke, On the existence of invariant measures for transformations with strictly turbulent trajectories, Bull. Polish Acad. Sci.

[15] A. Lasota and G. Pianigiani, Invariant measures on topological spaces, Boll. Un. Matem. Ital. (5) 14B (1977), 592-603. 
[16] G. Pianigiani and J. A. Yorke, Expanding maps on sets which are almost invariant: decay and chaos, preprint.

[17] W. H. Gottschalk and G. A. Hedlund, Topological Dynamics, Amer. Math. Soc. Colloq. Publ. Vol. 36, 1955.

[18] A. N. SharkovskiI, Coexistence of cycles of a continuous mapping of the line into itself, (Russian), Ukrain. Math. J. 16, no. 1 (1964), 61-71.

UNIVERSITY OF MARYLAND DEPARTMENT OF MATHEMATICS Collegge Park MARYLAND 20742 U.S.A.
AND UNIVERSITY OF MARYLAND Institute of Physical Science and Technology AND Department of Mathematics College Park MARYLAND 20742 U.S.A. 